\title{
Horizons/Théâtre
}

Revue d'études théâtrales

\section{Kossi Efoui, Dieudonné Niangouna et Bill Kouélany : la figure du trickster au cœur du geste d'écriture et de sa traduction scénique}

\section{Diane Chavelet}

\section{(2) OpenEdition Journals \\ Édition électronique \\ URL : https://journals.openedition.org/ht/1299 \\ DOI : $10.4000 /$ ht. 1299 \\ ISSN : 2678-5420 \\ Éditeur \\ Presses universitaires de Bordeaux}

\section{Édition imprimée}

Date de publication : 1 janvier 2019

Pagination : 10-27

ISBN : 979-10-300-0718-3

ISSN : 2261-4591

Référence électronique

Diane Chavelet, « Kossi Efoui, Dieudonné Niangouna et Bill Kouélany : la figure du trickster au cœur du geste d'écriture et de sa traduction scénique », Horizons/Théâtre [En ligne], 14 | 2019, mis en ligne le 01 juin 2022, consulté le 29 juin 2022. URL : http://journals.openedition.org/ht/1299 ; DOI : https:// doi.org/10.4000/ht.1299

\section{c) $(7) \Theta$}

La revue Horizons/Théâtre est mise à disposition selon les termes de la Licence Creative Commons Attribution - Pas d'Utilisation Commerciale - Pas de Modification 4.0 International. 


\section{Diane Chavelet}

Diane Chavelet est doctorante en littérature comparée à l'université Paris VII-Diderot, rattachée au laboratoire CERILAC, ED 131. Elle conduit une thèse intitulée : "La parole délivrée. Oralisation, performance et circulation du texte dans les arts dramatiques d'Afrique subsaharienne de langue française ultra-contemporains : 2000-2016. Autour de Dieudonné Niangouna (CongoBrazzaville), Bill Kouélany (Congo-Brazzaville), Kossi Efoui (Togo) » sous la direction de Catherine Coquio depuis 2014. Dans ce cadre, elle réalise en 2015 à Brazzaville un film documentaire sur le festival Mantsina-sur-scène, dirigé par Dieudonné Niangouna, Sommes-nous sortis du monde? Elle collabore à l'édition de la revue Travaux en Cours de Paris VII-Diderot en 2014 et contribue à la revue Po\&sie en 2016. Elle codirige la compagnie « Paupière Mobiles» et collabore à la mise en scène de deux pièces du dramaturge Guinéen Hakim Bah.

Mail : dianechavelet@yahoo.fr

Résumé: Le mythe du Trickster a alimenté depuis 1958, avec l'ouvrage de Jung, Radin et Kérényi qui référençaient un "fripon divin " dans la littérature orale amérindienne, une histoire critique qui dépasse l'anthropologie, et a intéressé les études littéraires postcoloniales et la philosophie politique avant d'être pensé dans une approche globale de l'histoire de l'art. Dans le champ des arts dramatiques africains, la figure du trickster, expression d'un imaginaire mythique de la subversion et de la résistance politique, s'impose dans la relecture des postindépendances et la pensée de

Abstract : The trickster's myth, since the famous essay The Trickster: A Study in American Indian Mythology (1958) by Jung, Radin and Kereny, had fed a critical history that overcome by far anthropology and psychoanalysis. Indeed, postcolonial literary studies and political philosophy have taken the subject in account before it has been studied in a perspective of global art history. In the specific field of African performing arts, the trickster's figure remains the expression of a mythical imaginary of subversion and political resistance, that is nodal to think
I'hybridation identitaire. Nous proposons de revisiter le personnage mythique au travers des productions textuelles et scéniques de trois dramaturges représentatifs de la génération des « enfants terribles » ciblée par Sylvie Chalaye : Kossi Efoui (Togo), Dieudonné Niangouna (Congo-Brazzaville), Bill Kouélany (Congo-Brazzaville).

Mots-cLÉs : figure du Trickster, dramaturgies contemporaines, Afrique subsaharienne, Dieudonné Niangouna, Kossi Efoui, Bill Kouélany.

both post independencies and hybrid identity. We propose to reconsider this mythical character through the literary and performing productions of three contemporary sub-Saharan dramatists, who are part of «the terrible children » targeted by Sylvie Chalaye: Kossi Efoui (Togo) Dieudonné Niangouna (Congo-Brazzaville), Bill Koulénay (CongoBrazzaville).

KeYWORDS: trickster, contemporary dramatists, francophone sub-Saharan, Dieudonné Niangouna, Kossi Efoui, Bill Kouélany. 


\section{Kossi Efoui, Dieudonné Niangouna et Bill Kouélany : la figure du trickster au cœur du geste d'écriture et de sa traduction scénique}

AVANT D'INTÉRESSER LES CHAMPS DE LA THÉORIE LitTÉRAIRE, des arts dramatiques ou plastiques, le mythe du trickster ou du « fripon $»$, littéralement « celui qui joue des tours », a d'abord intrigué l'anthropologie, la psychanalyse, et « l'ethnographie », discipline dont les fondements idéologiques ont été interrogés par Jean-Loup Amselle ${ }^{1}$ entre autres. La question qu'ont posée Jung, Radin et Kérény ${ }^{2}$ au sujet du « fripon » recensé dans la littérature orale amérindienne révèle un réflexe hégémonique : tout en cherchant à identifier la figure mythique à ses pendants grecs (Hermès ou Prométhée, Kérény), ils ont aussitôt cherché à comprendre comment « coexistaient tant de traits contradictoires en un seul personnage ${ }^{3} \gg$. Les réponses qu'ils apportent tendent plus à rationaliser le mythe dans le but de l'assimiler à une pensée « archaïque » résiduelle dans le sujet « civilisé » que d'envisager un mode de représentations basé sur d'autres fondements que ceux « des civilisations occidentales plus avancées ${ }^{4}$. »

Car la lecture rationaliste des mythes grecs ou bibliques postule qu'un personnage actant de tout récit génésique doit s'intégrer au sein d'une logique dualiste qui induit la « différenciation du bien et du mal », auquel le trickster paraît « préexister » (ibid.). Or le premier acte de subversion du personnage fripon est de miner toute représentation manichéenne des origines pour incarner une dualité organique. Lorsquà la fin des années 1980, le chercheur Henry Louis Gates reprend la question du trickster à la lumière du mythe Yoruba de Esu-Elegbara ${ }^{5}$, c'est pour comprendre précisément comment s'articule la notion de « double discours » dans la littérature afro-américaine. Bien que les récits mythiques divergent d'un peuple à l'autre, il apparaît qu'en Afrique de l'Ouest et centrale, les canevas se recoupent, du récit cosmogonique à la fable populaire qui en découle. Le trickster, qu'il soit nommé Esu-Elegbara (Yoruba), Legba (Adza-Tado regroupant les Fon, les Ewé) ou Ogo (Dogons), qu'il soit identifié à un renard pâle ${ }^{6}$, à un singe (Louis Gates) ou à un serpent arc-en-ciel (en Haïti), est la figure de la connaissance et de la transgression. Présent aux origines du monde comme l'une des premières 
créatures de Dieu, il incarne l'instance dissidente qui va participer à la création contre l'avis de son créateur. Dans la plupart des mythes, cette transgression s'accompagne d'un acte incestueux, car il lui faut féconder la terre, sa propre mère, pour en extraire la «graine » (le logos). Cet inceste entraîne un désordre cosmique qui aboutit à la création d'un monde incomplet et condamne le trickster à une forme d'errance, entre dieux et hommes, à la recherche d'une jumelle promise que le créateur lui soustrait à jamais. Médiateur entre l'humain et le divin sans être tout à fait l'un ni l'autre, c'est lui qui transmet aux hommes la connaissance d'une parole sacrée, dont découlent les pratiques de divination sous formes d'écritures géomantiques (Yoruba, Fon, Ewé). Dieu des « carrefours » car de la contingence, il protège et guide les voyageurs.

Parce que cette figure est le symbole d'une subversion de l'ordre établi, elle devient avec Louis Gates le socle d'une théorie littéraire qui entérine la dimension poético-politique des textes produits par les écrivains américains issus de la diaspora. Dans une autre mesure, la figure du trickster inspire au début des années 2000 la philosophie de l'art et politique ${ }^{7}$ (Belhaj Kacem : 2002), puis à partir de 2010, elle est de plus en plus pensée dans une relecture de la figure de l'artiste et dans une perspective globale ${ }^{8}$.

Dans un tel contexte critique, nous proposons d'interroger la pertinence de la figure du trickster à la lumière d'un renouveau des écritures dramatiques en Afrique subsaharienne. Kossi Efoui (Togo), Dieudonné Niangouna (Congo-Brazzaville) et Bill Kouélany (Congo-Brazzaville), ont en commun d'être apparentés, de fait ou par affinité en ce qui concerne Kouélany, à la génération « des enfants terribles », pour reprendre l'expression d'Efoui, ciblée par Sylvie Chalaye $e^{9}$. À rebours d'un théâtre d'intervention ou d'un « retour aux sources $\gg$ caractéristique des dramaturges qui les précèdent, et aussi dans la continuité du Congolais Sony Labou Tansi, ils interrogent l'identité et le contemporain au prisme de personnages hybrides, erratiques, qui rappellent par leur pouvoir de subversion le trickster mythique. Kossi Efoui revisite la figure pour recréer un lien entre le sacré et le vernaculaire, le politique et le poétique dans des contextes de dictature ou d'émergence de la pensée trans-humaniste. Dieudonné Niangouna fait du héros fripon un boxeur éclairé qui symbolise une résistance artistique dans un contexte d'aporie culturelle en Afrique subsaharienne, et met à jour les ambiguités inhérentes à la réception du «théâtre africain » en France. Bill Kouélany s'identifie, dans l'autobiographie fleuve qu'elle met en scène, à une créature hybride dont le travestissement est la marque d'une volonté de transgression inassouvie. 


\section{Kossi Efoui au « carrefour » de l'Histoire : le trickster au cœur du démantèlement des discours totalitaires}

Ce «carrefour », emblématique de la rupture qu’a incarnée Kossi Efoui avec sa première pièce éponyme ${ }^{10}$, qu'entérine-t-il ? Un non-lieu d'abord, « espace qui ne peut se définir ni comme identitaire, ni comme relationnel, ni comme historique ${ }^{11} \gg$ selon Marc Augé, celui de la scène dans le théâtre, éclairée par deux réverbères. Ils fonctionnent comme des projecteurs et « donnent vie » aux personnages qui se rencontrent à l'intersection symbolique, tous « revenants ». Un « poète », rendu à l'errance pour avoir chanté ses poèmes. Une « femme » délaissée dans l'entre-deux en l'attendant. Et un « flic », aimanté par cet endroit instable, parce qu'il est avant tout le lieu de la transgression, qu'il chasse. Par le procédé du théâtre dans le théâtre, tout est dit et joué d'avance : le non-lieu héberge un non-récit. «La femme » aime le « poète » et rejoue avec lui une romance impossible. Le «flic » chasse le « poète » qui se défend par sa parole. La «femme » se donne au «flic » pour sauver, sans succès, son amour. L'espace du trickster est un «nulle part » coincé entre les projecteurs, entre deux frontières artificielles, entre le royaume des vivants et le royaume des morts. Le trickster lui-même est un « souffleur », c'est-à-dire doublement celui qui détient le souffle et celui qui souffle le texte - ce double-discours qui se dit à tâtons. Le supplice qu'il endure et dévoile au terme de la pièce prend les dimensions d'une damnation mythique :

Le souffleur : 20 ans à l'ombre. Dans cette prison où tout disparaît. Où il ne reste que la mémoire. Répétitive. La mémoire détraquée, oublieuse, à qui je souffle, souffle des mots, des images qu'il faut sauver de l'amnésie. (98)

Car la parole poétique s'articule à reculons, toujours balbutiante à tenter de recoudre les lambeaux d'une mémoire éparpillée entre le rêve et le souvenir, l'Histoire qui se répète et le passé qui ne passe pas :

La femme : Et c'est pourtant la première fois que ça m'arrive. Comme une foule de choses qui ne m'arrivent qu'une fois mais qui me donnent l'impression d'arriver sept fois... Quand mon amie Rachel est morte, j'ai tout vécu, j'ai tout revécu exactement comme aujourd'hui. Comme quelque chose qui recommence. Cette douleur-là ça rend dure la gorge comme une boule de nausée et les yeux comme... comme... Non. Ça ne fait même plus mal, une douleur comme celle-là... ça ne blesse même plus ${ }^{12}$.

Cette parole hésitante dans un corps mutilé, réfléchie par le dispositif artificiel dans lequel elle surgit, cherche sans arrêt à se re-posséder, et ne se 
trouve vraiment que par analogies, fulgurances tropiques, chants, eux-mêmes éparpillés dans la reconstruction d'un mythe bafoué. Celui d'une « espèce en perdition, celle des voyageurs, des explorateurs, des curieux ${ }^{13} \gg$, qui se dresse, déjà vaincue mais toujours insistante, contre un ordre établi dans lequel elle n'a pas sa place, et par conséquent est menacée de destruction. Une guerre des discours est alors mise en œuvre dans l'espace scénique, lui-même artificialisé.

Le flic: Non. Je ne comprends rien à votre texte. Le seul texte qu'on m'a donné, c'est la loi. Parce que là tout est résumé en deux mots : tu arrêteras et tu conditionneras. [...] C'est-à-dire préparer un suspect à collaborer pendant l'interrogatoire $[. .$.$] On le démolit un peu à la limite de l'irréparable, on l'affame, on le$ prive de sommeil. C'est cela, conditionner ${ }^{14}$.

L'ordre établi qui rejoint sous la plume d'Efoui l'«ordre du discours 》 foucaldien possède des personnages-pantins, ici reflets allégoriques de régimes autoritaires, qui obéissent à l'injonction de « démolir » toute parole qui paraittrait faire « désordre ». La brutalité avec laquelle l'ordre légitime la destruction d'une parole déviante tient justement en ce que cette parole ne rentre pas dans le « texte » : ici référant doublement au texte de théâtre et au texte de loi, pour souligner par analogie l'artifice dans lequel agit ce dernier. Les corps marionnettiques incarnés par les trois protagonistes ne sont pas les mêmes : d'un côté «l'ordre du discours » affiche une transparence menaçante, et produit des machines «conditionnées » à neutraliser ceux qui formulent la «déviation ». De l'autre des corps brisés par un ordre suffocant sont réarticulés par un trickster afin de perpétuer la trace du vivant, c'està-dire de l'instable. Au discours du «flic » s'oppose la voix du «poète », subversive en ce qu'elle exhume la mémoire cachée des victimes d'un ordre totalitaire, mais aussi par son épaisseur tropique.

Le poète : Suspect. Encore ce mot. Au temps de mon ami, le peintre. Car il était classé suspect, lui aussi. Parce qu'il dessinait des images qu'on ne comprenait pas toujours. Alors de temps en temps les flics faisaient une descente chez lui et perquisitionnaient. Ils déchiraient ses livres et ses cahiers et ils brisaient ses tableaux. Et comme ils ne trouvaient toujours rien, ils l'emmenaient et le brisaient en petits morceaux pour perquisitionner en lui. [...] Un jour il est revenu. Et dans les restes de lui-même qu'il a rapportés et qu'il recollait, il n'a plus retrouvé sa langue. Et c'est fini. Il ne pourra plus jamais dire un mot. Un seul mot. Il ne peut que sourire ${ }^{15}$.

Si au carrefour menaçant de l'Histoire Kossi Efoui fait s'affronter l'ordre du discours et le poète dans un match joué d'avance où l'un a la force et l'autre 
la langue, ce qui fait échapper le poète - et le peintre - au fatum autoritariste, c'est son «sourire ». Même démuni de la faculté de voir ou d'articuler une parole, même réduit à ses propres morceaux, le vivant oppose encore au dispositif qui le détruit une ironie tenace et salvatrice, car suggestive d'une mémoire de la résistance, pas tout à fait enfouie.

Si Kossi Efoui confronte souvent dans ses pièces le discours totalitaire et la parole poétique, il en fait varier les formes. Les différentes mises en scène de ses pièces, prises en charge par Nicolas Saelens avec lequel il est en compagnonnage depuis 2004, travaillent la marionnette, réification de réminiscences, qui surgit au milieu de corps désarticulés par la pesanteur d'injonctions discursives. Dans En guise de divertissement (2013), la société du divertissement est représentée par des « histrions », acteurs à l'avant-scène qui tâchent de répondre à l'injonction de plaire par des démonstrations acrobatiques et des clowneries d'automates. Le spectre d'un Prométhée revisité, une grande marionnette anthropomorphe nommée «L'oiseau », vient perturber leur représentation pour exhumer une parole trouble, celle de la mémoire : «vos morts ne sont pas vraiment morts. » Les « histrions » se précipitent pour étouffer la créature et l'empêcher de poursuivre son discours. Le procédé encore une fois exploité du théâtre dans le théâtre permet de mettre à jour de façon significative une société dans laquelle le divertissement tient place d'un masque utile à cacher ce qui la gangrène. Ainsi le caractère asphyxiant des discours institués est révélé dans les pièces d'Efoui par une mise en abyme de sa représentation, et par sa confrontation à d'autres voix enfouies, car perturbatrices, qui rappellent une humanité oubliée. Dans Sans ombre. Une satire transhumaniste ${ }^{16}$ (2017), la compagnie Théâtre Inutile, accompagnée $\mathrm{du}$ philosophe Miguel Benasayag, s'intéresse à la progression du discours transhumaniste qui semble dénier le vivant au profit de la techno-science. Le texte de Kossi Efoui s'ouvre sur la propagande marketing de «Strong Life United $\gg$, une société qui vend un monde virtuel où l'univers entier semble «modélisé », pour permettre aux hommes d'échapper à la « catastrophe » écologique, de « tuer la mort » et de faire partie de la « nouvelle espèce, pour une somme de trois millions $\gg$. Derrière l'utopie progressiste, mise ici au service d'une propagande consumériste, se dévoile bientôt un argumentaire fondé sur une vision téléologique de l'Histoire, « le dépassement de la Nature par l'homme $\gg$, qui réinvente dans un récit aux accents épiques les conquêtes de Christophe Colomb et de Vasco de Gama. La « Vox ex machina », narratrice mécanique du mythe rationaliste, apostrophe le public pour décrire les terres conquises alors peuplées de « ces hommes extrêmement noirs, ces hommes extrêmement bruns, ces habitants des côtes sauvages où la nature 
est reine $[\ldots]$ ces hommes prisonniers du temps cyclique $\gg^{17}$ et qui, grâce aux explorateurs-civilisateurs, « virent venir à eux le temps de l'Histoire ». Cette «représentation, offerte par Strong Life United», permet d'établir la continuité entre le discours colonialiste et celui de la techno-science, laquelle perpétue dans son argument même l'idéologie qui a légitimé l'asservissement des peuples « indigènes ». Car à partir de la colonisation, selon Henri le Navigateur, seront différenciés les « hommes réels $\gg$ des $\ll$ hommes imaginaires », les premiers «ponctuels dans le même temps présent, celui des horloges, celui de l'Histoire » et les seconds « condamnés » à l'oubli. Dès lors l'asservissement de l'homme à la technique (l'horloge) détermine l'appartenance ou non des peuples à l'espèce humaine. Deux perturbateurs interviennent dans la « représentation » de «Strong Life United », chacun miroirs inversé de l'autre : Polichinelle et Wilderman. Polichinelle représente le bouffon, la face profane du trickster, qui joue à caricaturer les intervenants, à piéger le décor. Wilderman représente sa face sacrée, autre Prométhée qui porte en lui l'«Esprit de toute chose $»$. Venu au monde avec un message pour l'homme, il « ne le reconnait pas », et s'adresse donc à Polichinelle sous couvert d'être l'inventeur « Thomas Edison », puis « Prométhée »:

Wildermann : [...] Je dis : Ce que nous savons, c'est que nous ne savons rien... Si nous ne connaissons pas la millionième partie des choses de ce monde, si nous ignorons ce que c'est au juste l'eau, au juste la lumière, au juste la gravitation, au juste l'électricité, au juste la chaleur... je dis : si nous ne savons rien au juste du magnétisme, comment pourrions-nous savoir au juste ce qui se passe dans l'invisible? (SA : 2017 ; p. 15) [...] C'est la voix de Prométhée. Celui dont les machines tiennent leur pouvoir, même si elles ne le savent pas. Dans le feu de Prométhée, les rayons sont innombrables : le rayon de la science, le rayon de la poésie, le rayon de la guérison, le rayon de la divination pour connaître l'avenir... Polichinelle : Plus besoin d'avenir. Wildermann : Le rayon des arts pour percevoir dans l'Invisible. Polichinelle (parodiant) : Demain, bientôt, très vite La terre, la Nature et ce qu'on appelle encore l'homme rejoindront le Royaume des fantômes, des chimères, de l'imagination, de la fiction, de la fable. Bienvenu dans un monde sans ombre. (SA : 2017 ; p. 21)

Au discours de propagande asséné par «Strong Life United» par des machines et des hommes qui leur ressemblent, et caractérisé par l'anaphore, l'assertion et l'apostrophe, la marionnette prométhéenne oppose des questions rhétoriques posées dans un style archaïque qui rappelle également la dialogique socratique. La répétition de l'expression « au juste » suggère une mise en question de ce que le discours transhumaniste qualifie de « réel », tandis que la limite du « savoir » humain vient ici contrefaire sa volonté de 
puissance. L'effet de solennité biblique produit par l'introductive « je dis » accentue la portée sacrée du discours.

Le trickster rappelle aux hommes-machines une organicité oubliée avec les « horloges », celle d'un univers fait d'ombres, d'« invisible » que la technosience est impuissante à recréer dans un monde «virtuel » doublement faussé par sa mimesis et le discours téléologique sur lequel il s'est créé. L'espace scénique, non-lieu de la représentation, permet la déconstruction du mythe occidental par une mise en abyme qui en découvre l'artificialité. La marionnette tient ici le rôle du lien sacré entre l'homme et son «ombre », libère une parole qui ouvre au poétique, lorsque les hommes-machines recrachent un discours qui vise à l'anéantir. Les « rayons innombrables » de Prométhée rappellent ainsi que la technique antique transmise aux hommes leur permet de comprendre, et non d'asservir, la « Nature », «l'Invisible » ou l'《avenir ». Dans le monde «sans ombre » promis par «Strong Life United », c'est un présent virtuel qui est vendu au sacrifice de la mémoire et au mépris de l'avenir, projet qui promet de «modéliser » les hommes, et par là même de leur ôter toute humanité car toute possibilité de libre arbitre. Le trickster efouien fait ici le lien entre le sacré (l'écriture du commencement du monde) et le vernaculaire (l'appropriation de son destin par l'homme), la permanence (des éléments) et le mouvement (de l'organique). Il incarne ainsi la mémoire de l'inutile qui préside à l'organisation du vivant, et fait ainsi faillir la visée destructrice du projet trans-humaniste.

\section{Dieudonné Niangouna : boxer avec son ombre pour une poétique de la résistance}

C'est dans le non-lieu de l'hôpital, « avec des murs tout blancs et des fils de fer barbelés tout noirs » que Dieudonné Niangouna libère la parole du «Clando ». La mise en scène de son texte, Attitude Clando, qu'il donne à Avignon en 2007 souligne la dimension sacrificielle d'un non-lieu : un cercle de cailloux délimite l'espace scénique, qui devient lui aussi un carrefour, puisqu'il ne représente aucun lieu-dit et n'existe que le temps du rituel de la représentation. Le destinataire de cette parole est un «docteur» invisible mais qui personnifie l'institution neutralisante dans laquelle le personnage est « coincé », tout comme le « souffleur » d'Efoui est condamné à ressaisir la mémoire « détraquée » dans sa « prison ». À l'instar des protagonistes du Carrefour, le Clando de Niangouna se dérobe à l'ordre institutionnel dans la forme même d'un discours décousu qui atteste de son inconformité, jugée par l'institution qui l'enferme comme une irrégularité : 
Suis hors pair, hors marge ni dans la page, hors périmètre, suis pas à la surface, inachevé, fort à mon aise. Je ne pue même pas le tordeur de la loi. Mais le rien à foutre. Tordre la loi c'est des idées comme tuer quelqu'un. Moi j'ai pas d'idées. Je cherche du piment ${ }^{18}$.

L'adjectif « inachevé » convient bien à la langue du personnage, rythmée par des procédés de parataxe et d'asyndète qui attestent de son urgence, et justifient son économie tout en sculptant sa musicalité. La figure du trickster se déploie ici dans son irrévérence et son inquiétude, car le Clando compte sur sa propre logorrhée pour sortir d'un «périmètre » qui l'accule à une normalisation mortifère. L'absence de réponse du docteur, aussi invisible qu'impassible, fait écho « aux murs blancs » que le Clando ne parvient pas à franchir. La violence que l'institution lui oppose est moins directe que celle infligée métaphoriquement au « peintre » dans la pièce d'Efoui. Mais elle est tout aussi brutale. Car Niangouna souligne une nuance bien esquissée chez Efoui entre l'ordre social et l'ordre totalitaire : dans l'un le « coupable » est celui qui enfreint la loi par son crime, dans l'autre, le « suspect $\gg$ est celui qui enfreint le discours politique par sa parole. Le premier «symptôme », pour rester dans un lexique clinique, de cette esquive à l'ordre totalitaire, réside donc dans la langue même, qui s'écorche à la mesure d'une subjectivité brimée. Le protagoniste de Niangouna n'est pour autant pas un artiste, mais il est dépositaire d'une mémoire trouée, et à la recherche de cette mémoire, pays perdu où pousse le « piment », c'est-à-dire « une tentative de vie » :

Ma maman m'avait planté un pimentier dans la cour de notre maison, là-bas, au pays, à l'époque où cétait encore un pays. Personne n'osait toucher ce pimentier, il était d'une nervosité correcte, moi, il me reniflait tout amicalement, il caressait ma langue et attendrissait mes nerfs : c'est ça mon pays. Mon pays c'est le piment. Et maintenant je ne peux plus envoyer d'argent au pays parce qu'ils ont coupé mon pimentier. Ça m'a saoulé. Couper mon pays c'est me tuer. J'ai pris mon sac au dos, puisque j'étais mort, et je suis parti ${ }^{19}$.

La relation gémellaire entre le «Clando » et le «pimentier », synecdoque $\mathrm{du}$ « pays », se construit sur un procédé de personnification du végétal, amorcée par la main de la mère qui lui donne symboliquement vie. La «nervosité correcte » de l'arbre traduit son lien à la racine et a pour effet d'enraciner aussi le protagoniste. La contamination nerveuse de l'arbre à l'homme est rendue par le polyptote «nervosité », «nerfs ». Dès lors, et dans la suite logique du cheminement analogique, « couper le pimentier », c'est « couper le pays » et équivaut pour le «clandestin» à le «tuer ». Seule la parole, rendue performative par le dispositif scénique qui en souligne 
la dimension expiatoire, « enracine » le sujet dans l'instant de profération. Symbole de l'enfermement du sujet et de la libération de sa parole, le carrefour devient donc lieu de résistance, l'espace dans lequel se joue une impasse dont la représentation même tend à l'en faire sortir.

Car c'est bien le rôle que Niangouna, avec Efoui, semble donner à la réécriture du mythe du trickster. Le fatum qui s'est abattu sur son personnage est celui, non des dieux, mais de l'Histoire. Réactiver un schème mythique dans la composition dramatique n'équivaut pas dans ce contexte à nier l'historicité du récit, mais à mettre en perspective une continuité vertigineuse. À cette pesanteur mnésique, qui semble agir comme un implacable déterminisme et figer les êtres dans leurs archétypes (《Le poète » maudit, le «Clandestin » condamné) la figure du trickster oppose une résistance par la parole. Une parole qui, par son épaisseur poétique et par sa destination scénique, devient performative car elle introduit le chaos dans l'espace oublié du carrefour, et ouvre ainsi une brèche dans la construction lisse d'une violence discursive : ce «mur » clinique sur lequel le Clando tape sa tête. Une parole qui extrait de l'archétype une singularité, qui déboute la permanence historique pour y insuffler de l'intime.

Le monologue du «Clando » s'achève sur un « coup de feu » qui suspend sa parole et ferme le cycle rituel : le sacrifice a eu lieu. Mais les mots qui précèdent le suicide symbolique entrent en résonance avec les textes qui vont suivre, et marquent chez Niangouna les prémices de manifestes poético-politiques :

Nous sommes hors terre. Ce que tout le monde oublie et que moi seul je sais, c'est qu'il y a dans l'espace des milliards de grosses pierres qui filent plus que la lumière. [...] Et c'est jamais toi qui les évites. Mais il y a en toi un sens de parer les coups qui va plus vite que tes nerfs, que ton instinct animal, que ta pensée fertile, plus vite que la frappe du soleil. [...] Parce que cette chose là qui feinte coup sur coup n'est pas localisée en toi. Personne ne l'a nommé. Il ne figure dans aucune biologie des sens. C'est un Clando. Jamais en place, jamais en règles ${ }^{20}$.

La référence à un désordre cosmique dans lequel un « clando » ontologique éviterait les coups de « grosses pierres qui filent plus que la lumière » place la spécificité de ce trickster au-dessus d'un déterminisme social, pour esquisser une poétique de la résistance. C'est bien une « attitude » qui est ici revendiquée et non la complainte d'un individu assigné à l'errance par l'ordre géopolitique et à l'isolement par l'ordre médical. Cette praxis de la « feinte coup sur coup $\gg$, qui emprunte déjà ici le lexique du sport de combat devient dans M'appelle Mohamed $\mathrm{Ali}^{21}$ le mot d'ordre d'une résistance à une hégémo- 
nie culturelle et symbolique héritée du colonialisme, par le développement du théâtre en Afrique.

En 2014 est portée à la scène la pièce écrite par Dieudonné Niangouna pour le comédien burkinabé et directeur du festival «Les Récréatrâles », par Dieudonné Niangouna. Elle fera le tour du continent africain et de toutes les grandes salles européennes affiliées à la «francophonie ». M'appelle Mohamed Ali est construite sur la mise en abyme d'une pièce de théâtre dans laquelle Étienne Minoungou interprète le célèbre boxeur lors de son dernier combat, quasi-mortel, avec Frazier, le $1^{\text {er }}$ octobre 1975, à Manille. Ce flirt avec la mort est prétexte aux réminiscences d'une vie, marquée par les luttes physique et politique. Le cheminement dans l'histoire de Mohammed Ali est aussi prétexte aux digressions d'Étienne Minoungou, qui sort de la peau du boxeur, double fantomatique, pour questionner ses propres luttes. Le texte oralisé est construit pour générer la confusion quant à une intention délibérée de l'acteur à sortir de son personnage pour entrer en dialogue avec le public. Ainsi la pièce retrace-t-elle l'historiographie d'un «combat avec la mort $\gg$, les enjeux de la ségrégation aux États-Unis et le refus de soumission au gouvernement américain, emblématisée dans la figure du boxeur dans les années soixante-dix, déplacés par l'acteur à l'impuissance d'exister en tant que dramaturge africain en Europe et la lutte solitaire pour rendre possible un théâtre en Afrique aujourd'hui. Joute cantonnée, dans la veine césairienne, à une métaphore trop strictement virile mais dont l'efficacité fait aussi la force du spectacle. M'Appelle Mohamed Ali renferme en réalité, et à plusieurs niveaux, des enjeux stratégiques. Dissociant clairement les préjugés d'un spectateur occidental non-averti et les enjeux contemporains de politiques culturelles inhibant la production artistique africaine, le lien tissé avec le public participe d'une progression rhétorique habile. Entrer dans la peau de Mohammed Ali permet à la fois à l'acteur de prendre ses distances par rapport à des revendications de reconnaissance identitaires aujourd'hui jugées obsolètes, et de mettre en lumière la pérennité de certains de ces comportements sociaux-politiques - particulièrement dans le cas du retard français sur ces questions. Même enjeu pour ce qui est de la critique du chantage du gouvernement américain dans le cas précis du procès de Mohammed Ali, qui devient le reflet d'une politique culturelle n'accordant de visibilité aux artistes qu'à condition de les travestir. Ce qui permet finalement de marier boxe et théâtre, le style si particulier de Mohammed Ali accolé à celui du dramaturge souvent jugé hermétique, comme deux armes aux fins d'une même résistance, chacun faisant sienne dans la discipline qu'il pratique une poétique de l'esquive qui rappelle les qualités du fripon mythique : 
Faut jamais devenir une simplicité à définir pour l'autre. Un enjeu parfait à comprendre par l'autre. «Danser. »Insaisissable, c'est l'art de ne pas te laisser accaparer. Souffle comme le vent. Reste souple à chaque matière. La performance est de livrer un combat au-delà de l'endurance physique, au-delà des frontières de soi et au-delà de toutes les attentes. [... ] L'art de boxer est une loyauté qui nous enseigne qu'on peut lutter contre, combattre et vaincre toutes les impossibilités de l'univers. Et pour faire du théâtre en Afrique il faut boxer la situation. Mais vous ne savez pas ce que je suis de mon théâtre et ce qui, en moi, fait rectifier les écarts et brûler les attendus. L'histoire que vous attendez n'aura pas lieu. Vos attendus l'ont déjà mangée. C'est ça mon théâtre. Mon théâtre est le drame de ce qu'on veut du théâtre africain. Le ring est un dialogue, et la scène un champ de bataille. [...] Mohamed Ali fait partie de ces rêves que j'ai créés tout le temps, que le monde m'a vu dégringoler les dieux un à un de leur montagne du Caucase pour aller libérer Prométhée $e^{22}$.

Nous percevons bien comment ce triangle énonciatif (Étienne Minoungou parle à travers Mohamed Ali et Dieudonné Niangouna à travers Étienne Minoungou) a fonction de mettre en abyme le topos du combat de boxe dans une lutte qui excède le champ théâtral africain et embrasse l'histoire des contre-pouvoirs. Le théâtre devient le médium d'une pensée de la résistance qui s'articule autour de la « performance », la scène et le ring étant chaque fois le lieu d'un combat mené par une stratégie de l'esquive. Si dans ce cas-là le récit est historicisé, il n'en revoie pas moins à des schèmes cosmiques et mythiques qui structurent une pensée dissipative caractéristique de la figure du trickster.

\section{Bill Kouélany : d'une marginalité littéraire à l'éclatement scénique}

Dans son premier récit allégorique, Vagabondages ${ }^{23}$, Bill Kouélany replace également le mythe du trickster dans un présent indéfini pour réactiver sa dimension politique. Une « vagabonde » arrive dans un village situé au « Pays Congo $\gg$, et se heurte à la méfiance des femmes, au désir des hommes. Elle vit plusieurs aventures, dont une homosexuelle, avant de rejoindre l'insouciance d'un groupe d'enfants mené par un personnage du nom de « Pierre-yaya ». Le dernier chapitre du récit de Kouélany propose la mise en abyme d'un conte génésique. « Pierre-yaya », entouré d'enfants au centre (au carrefour) du village, raconte l'histoire d'un dieu hermaphrodite qui, par la peur qu'il inspire aux hommes « de la race des Il-faut-être-réaliste et des Deux-fessesbien-assises-au-sommet-de-la-hiérarchie-sociale », sera vite affublé d'un « sexe faible » et du nom de « mâ Nkanka, la vieille des vieilles, pour limiter les possibilités qu'ils redoutaient tant, pour l'enfermer dans un espace donné, bien connu d'eux, pour mieux la contrôler $\gg$ (p. 58). Afin de ne pas s'en atti- 
rer les foudres, les hommes lui offrent les relégués de la société, « Hommesenfants », que « mâ Nakanka » emmène dans son royaume vivre aux côtés de sa fille «Imagination », «atypique, aérienne, libre, fantasque, folle ». $\mathrm{Du} \ll$ vagabondage » des Hommes-enfants avec Imagination naissent des « œuvres à secouer le monde », ce qui n'est pas du goût des hommes aux «Deux-fesses-bien-assises-en-haut-de-la-hierarchie-sociale». Le mythe s'achève par une explication métaphorique des origines de la «monnaie » :

Craignant la dégringolade pour leurs belles fesses bouffies mais si fragiles, mais surtout jaloux du succès de ces êtres dont la réputation traversait les frontières, ils décidèrent de tuer tous enfants en l'homme. L'uniformité devint de rigueur; à tout humain, ils imposèrent la monnaie pour avoir droit au manioc. Et pour avoir cette monnaie il fallait travailler, travailler, travailler... Pas de monnaie, pas de manioc, pas de manioc pas de rêve, pas de rêve... ${ }^{24}$

Nous voyons bien ici comment la réécriture du mythe induit une lecture de l'histoire coloniale et postcoloniale : si l'espace du récit est celui du « Pays Congo $\gg$, dès lors l'introduction de la «monnaie » asservissante peut-être lue comme celle du Franc CFA, destinée à protéger aussi les instances de pouvoir politiques, anciens colonisateurs ou dictateurs en place. L'asservissement des populations au profit du maintien du pouvoir en place est résumé par la métaphore parlante du «meurtre de l'enfant » en l'homme par l'introduction de l'argent, qui l'affame jusqu'à le priver de « rêve » - et de la possibilité de créer, en réponse. Nous voyons aussi comment Kouélany imbrique la question du genre à une généalogie de l'ordre social : le Dieu est craint car il possède deux sexes, et le féminiser revient à en prendre le « contrôle ».

Mais le dénouement proposé par Kouélany, qui poursuit le mythe et clôt Vagabondage, a vocation de formuler un contre-mythe qui résonne comme un programme de résistance poétique. Car c'est la faculté de rêver que « Pierreyaya $\gg$ a rendu aux enfants qui l'écoutent.

Le récit de « Pierre yaya » est suspendu, en dépit des réclamations bruyantes de son auditoire. Au terme de cette initiation rituelle, où les enfants ont respecté l'ordre du récit, ont encouragé le conteur, ont ponctué l'histoire de leurs serments, « Pierre-yaya » impose un silence. Ils entrent alors dans une forme de transe qui les conduit chacun à adopter une forme d'art. L'un se met à danser et chanter du rap, l'autre à écrire, un autre à peindre, comme pour réitérer dans leur présent la trame du conte, et opposer encore au mythe des puissants la force d'une œuvre instable. Une instabilité qui puise aussi sa force dans l'inter-médialité, car ce n'est pas une discipline qui fait œuvre dans le dénouement de Vagabondage, mais le branchement simultané de plusieurs transports poé- 
tiques qui se choisissent une forme spontanée. En ce sens, Kouélany rejoint une conception de l'art comme « jeu $\gg$ formulée aussi par Belaj Kacem ${ }^{25}$. Le mythe du trickster, ici directement itératif dans la fable et par la fable, contient une trame dans laquelle s'imbriquent et s'opposent deux formes de genèses : celle de l'ordre et celle du désordre, ce dernier étant à la fois l'origine et la condition du geste artistique. L'art, première opposition au pouvoir politique dans l'ordre du mythe, n'est paradoxalement subversif que parce qu' il s'extrait radicalement du discours politique. Il n'oppose pas de « contre-discours », mais « un langage poétique $\gg$ au sens que donne Roland Barthes $^{26}$ à la recherche poétique qui lui est contemporaine. Le lieu de cette parole est toujours un « carrefour » : un non-lieu au sens de Marc Augé, ouvert à la contingence dont le geste artistique a l'exigence, et qu'entérine aussi l'espace scénique.

À bien des égards, Vagabondage tient une place programmatique dans l'œuvre de Kouélany. L'ouvrage qui suit, projet autobiographique jamais achevé sous le nom d'Extraits d'acte de naissance ${ }^{27}$, tient une parole amorcée dans le récit allégorique. Assumant un « je » qui n'a de cesse que de procéder à son autopsie, elle assume la part hybride de son propre personnage, mythifiant ainsi, par référence in-absentia au Dieu hermaphrodite dont Vagabondage fait le conte, l'identité artistique même. Assumer le transgenre, et commencer pour ce faire à choisir le prénom de Bill, c'est échapper à la volonté de « contrôle » de ceux qui se maintiennent au pouvoir dans l'ordre social, et commencer par là même à s'affranchir d'une injonction conformiste qui a pour effet d'inhiber la pulsion créatrice. Le mot «femme », quand elle ne parle pas de la femme des autres ou de celles qu'elle désire, est dans son texte toujours mis à distance ou au conditionnel, irréalisé et incompris. Sans doute car ce nom, affublé du verbe être, contient un projet de dépersonnalisation.

Si j'avais été une femme comme savent l'être des vraies femmes avec un vrai mari, j'aurais su accueillir dans ma chair ouverte sa chair tendue. Mais c'est peut-être le mot «femme »qu'au fond, je n'ai jamais compris. [...] Année 1983: Bilan; j'avais dix-huit ans, j'étais la femme d'un homme, j'avais un gosse. Autrement dit, rien ${ }^{28}$.

Construire une identité artistique revient dans Extrait d'actes de naissance à s'extraire de toute identité sociale. Ainsi la narratrice se définit-elle par la négative. Ni congolaise, ni française, elle s'assigne à un « nulle part », carrefour dans lequel nidifient à la fois l'isolement et son possible corollaire, la créativité :

Mes parents m'avaient envoyé en France dans l'espoir d'y revenir soigneusement lavée, lissée, polie. J'y revins comme Lazare après un séjour chez les morts, pour reprendre les mots de Jankélévitch parlant du récit de Léonid Andreïv sur Lazare, avec un je ne sais quoi, qui m'éloignera à jamais des autres. Pas vraiment ici ni 
tout à fait là-bas. Nulle part à jamais! (EAN, 35) On me jugeait hautaine, fière, méprisante, dure, je n'avais les pieds nulle part. Personne ne pouvait mesurer mon déracinement ${ }^{29}$.

La référence au retour de Lazare du royaume des morts, et particulièrement à la réécriture du mythe biblique par Jankélévitch (Quelque part dans l'inachevé : 1978), n’a ici rien d'anodin. L'isolement inhérent à l'expérience de l'exil, que Kouélany traduit en un exil social et intérieur, permet la rencontre et l'identification au peuple de l'entre-deux : celui de nombreux artistes, autant de tricksters sans doute, communauté fantomatique sans cesse convoquée par les livres et les œuvres, dont l'évocation fonctionne comme recréation d'un royaume mythique où des « hommes-enfants $\gg$ flirtent avec la belle « Imagination. » Ce territoire oublié de l'art - pris dans la conception bien singulière qu'elle lui accorde - héberge des hybridités identitaires qui endossent l'hybridation créatrice. La trajectoire de Kouélany atteste du désir de traduire une parole poétique en autant de médiums qui la réinventent et la réfléchissent. En atteste ses œuvres plastiques, qui jouent toujours avec le texte : ou pour traduire le poème en fulgurances visuelles ${ }^{30}$, ou pour déconstruire le discours institué dans un démantèlement plastique (collages de coupures de journaux). En atteste également sa mise en scène d'Extraits d'actes de naissance (2015), spectacle dans lequel elle fait correspondre des bribes autobiographiques avec le corps disloqué d'une circassienne, dont l'expression physique entre en échos avec des images projetées en fond de scène. Faisant s'entrechoquer plus que se mêler, cirque, danse, vidéo, musique et texte, Kouélany éclate le récit autobiographique dans un désir de « totalité inachevée », œuvre qui reconstitue le corps morcelé en une unité discordante pour répondre par un chaos artistique à la menace mortifère de la catastrophe politique ${ }^{31}$ :

Guerres. Guerre civile, guerre étrangère, guerre des sexes, guerre des sentiments, guerre des mots, guerre de l'argent, guerre des idéologies, guerre des races... Franchement, je m'en fou Ce qui compte, c'est ce que je fais avec. Ce que l'artiste fait avec.

Il n'y a que l'art. Il n'y a que l'instant de la création qui compte. Oh, oui que j'aimais ce chaos! [...] Mon art est une récupération de tout et de rien: patchwork de tout et de rien. Je crée avec tout ce qui croise mon chemin. Tout devient matière. Construction et reconstruction. Création et recréation. Je suis une maison cassée. Belle cassure. Bien debout ${ }^{32}$. 
Dans la relecture qu'en font Efoui, Niangouna et Kouélany, la figure du trickster n'est pas une construction mythique. Pas dans le sens accordé par Roland Barthes du moins, où le mythos se pense comme étroitement imbriqué à la propagande politique. Il est en réalité l'exact revers d'une telle conception, ou pour ainsi dire, le mythe du contre-mythe, le récit renversé des origines où apparaît sous une forme presque-humaine le créateur d'un autre discours qui par sa puissance poétique renverse l'ordre du monde.

En cela, les trois dramaturges s'accordent à donner au trickster les attributs d'une figure de la résistance à un « ordre du discours » qu'ils démantèlent dans l'espace scénique. Un non-lieu d'où progressivement émergent les lignes de fuite d'une autre Afrique, en gestation, ni prisonnière de son passé ni esclave de son avenir, promesse d'un continent qui oppose au chaos géopolitique la force d'un chaos créateur hybride et têtu, car,

Écrire, pour nous, signifie dans ce contexte que nous opérons la douloureuse descente aux enfers sans ceillères, prêts à tout assumer des désillusions présentes et futures. Il s'agit d'une esthétique du danger face au pouvoir inquisiteur des normes qui sanctionnent et censurent l'imagination créatrice ${ }^{33}$.

\section{Bibliographie}

Amselle Jean-Loup (dir), Le sauvage à la mode, Paris, Le Sycomore, 1979.

Augé Marc, Non-lieux : introduction à une anthropologie de la surmodernité, Paris, Seuil, 1992.

Barthes Roland, Mythologies, Paris, Seuil, 1957.

Belhaj Kacem Mehdi, La théorie du Trickster, Paris, Sens \& Tonka, 2002.

Chalaye Sylvie, «Les enfants terribles du théâtre francophone contemporain » in Africultures, 30 septembre 1997. URL : http://africultures.com/les-enfantsterribles-du-theatre-africain-francophone-contemporain/

Efoui Kossi, Récupérations, «Post-scriptum », Morlanwelz (Belgique), Lansman, 1992.

— Le Carrefour, in Théâtre Sud, nº 2, Paris, L'Harmattan, 1990.

- Sans ombre. Une satire transhumaniste, tapuscrit, 2017.

Griaule Marcel et Dieterlen Germaine, Le renard pâle. Histoire de la création de l'Univers par le peuple Dogon, Paris, Institut d'ethnologie, 1965 (1 $1^{\text {re }}$ édition).

Hyde Lewis, Trickster makes this world: Mischief, Myth, and Art, New York, Farrar, Straus and Giroux, 2010.

Jung C. G., Kérényi Charles, Radin Paul, Le fripon divin, trad. de l'allemand Arthur Reiss, Genève, Georg Éditeur, 1958 ; 1984, p. 145.

Kouélany Bill, Extraits d'actes de naissance : flux et fluences, tapuscrit, 2012. 
— Extraits d'actes de naissance, tapuscrit, 2004.

- Vagabondages, tapuscrit, 1990.

Louis Gates Jr. Henry, The signifying Monkey. À theory of Afro-American Literay Criticism. Oxford University Press, New York, USA, 1988.

Makarius Laura, "Le mythe du Trickster », Revue de l' histoire des religions, t. 175, $\mathrm{n}^{\circ} 1,1969$. p. 17-46.

Niangouna Dieudonné, M'appelle Mohamed Ali, Besançon, Les Solitaires Intempestifs, 2014.

- Les Inepties volantes, suivi de Attitude clando, Besançon, Les Solitaires Intempestifs, 2010.

\section{Notes}

1. J.-L. Amselle (dir.), Le sauvage à la mode, Paris, Le Sycomore, 1979.

2. Jung C. G., Kérényi Charles, Radin Paul, Le fripon divin, trad. de l'allemand par Arthur Reiss, Genève, Georg Éditeur, 1958 ; 1984.

3. Makarius Laura, "Le mythe du "Trickster" ", Revue de l'bistoire des religions, t. 175, $\mathrm{n}^{\circ} 1,1969$, p. 17-46, p. 18-19.

4. Jung C. G, Kérényi Charles, Radin Paul, Le fripon divin, Georg Éditeur, trad. de l'allemand Arthur Reiss, Genève, Suisse, 1958 ; 1984, p. 145.

5. Louis Gates Jr., Henry, The signifying Monkey. À theory of Afro-American Literay Criticism. Oxford University Press, New York, USA, 1988.

6. En référence à Griaule, Marcel et Dieterlen, Germaine, Le renard pâle. Histoire de la création de l'Univers par le peuple Dogon, Institut d'ethnologie, Paris, 1965 (1 $1^{\mathrm{re}}$ édition).

7. Belhaj Kacem, Mehdi, La théorie du Trickster, Paris, Sens \& Tonka, 2002.

8. Hyde, Lewis, Trickster makes this world: Mischief, Myth, and Art, Farrar, Straus and Giroux, New York, 2010.

9. Dès 1997, Sylvie Chalaye recense une nouvelle génération de dramaturges africains : voir l'article en ligne sur le site d'Africultures « Les enfants terribles du théâtre francophone contemporain » http://africultures.com/les-enfants-terribles-du-theatreafricain-francophone-contemporain/

10. Nous référerons pour cet article à la version de la pièce de Kossi Efoui, Le Carrefour, parue dans le $\mathrm{n}^{\circ} 2$ de la revue Théâtre Sud, Paris, L'Harmattan, 1990.

11. Augé, Marc, Non-lieux : introduction à une anthropologie de la surmodernité, Paris, Seuil, 1992, p. 100.

12. Kossi Efoui, Le Carrefour, in Théâtre Sud, n 2, Paris, L'Harmattan, 1990, p. 70.

13. Ibid., p. 71.

14. Ibid., p. 81-82.

15. Ibid., p. 78.

16. La pièce demeure inédite. Nous référons pour cet article à la dernière version du tapuscrit original qui nous a été transmis par l'auteur.

17. Kossi Efoui, Sans ombre. Une satire transhumaniste, tapuscrit, 2017, p. 8.

18. Niangouna, Dieudonné, Les Inepties volantes, suivi de Attitude clando, Besançon, Les Solitaires Intempestifs, 2010, p. 85. 
19. Ibid., p. 83.

20. Ibid., p. 93.

21. Niangouna, Dieudonné, M’appelle Mohamed Ali, Besançon, Les Solitaires Intempestifs, 2014.

22. Ibid., p. 46 ; p. 55-56.

23. Texte inédit - nous référons au tapuscrit daté de 1990.

24. Bill Kouélany, Vagabondages, tapuscrit, 1990, p. 58.

25. Belhaj Kacem, Mehdi, La théorie du Trickster, Sens \& Tonka, Paris, 2002, p. 16-17 : « Le jeu devient là plus critique : accomplissant en commun l'œuvre des corps, désormais abandonnés à leur détresse, à leur désœuvrement, il est l'art se contestant lui-même plus loin qu'il ne le fit jamais, et exigeant de lui-même plus qu'il n'exigera jamais. Ceux qui y prennent part, veulent les transports les plus risqués, les passions les plus vives. Il faut beaucoup d'art, pour effacer toutes traces de celui-ci, le plus grand art, pour l'indiscerner de la réalité des corps. [...] Le jeu devient la réalité des corps et ne semble plus être de l'art, et même plus de l'anti-art, cette marotte épuisée. Il est ce réel des corps, et ce réel aréal des passions, demandant leur dû à l'art, à ce dont l'art fut toujours le recel, l'expropriation. Et c'est par là qu'il perpétue ce que toujours l'art fut : sous prétexte de restituer les passions, les exproprier ; et, dans le mouvement inverse, qui est son devenir même, se réapproprier ce dont il fut, peu auparavant, l'expropriation. Qui joue, joue en artiste, avec sa langue, sa représentation. Mais comme jouer, c'est avec sa peur, son angoisse, qu'il éprouve l'art. »

26. Roland Barthes, Mythologies, Paris, Seuil, 1957, p. 243 : "Voici un autre langage qui résiste autant qu'il peut au mythe : notre langage poétique. [...] Alors que le mythe vise à une ultra-signification, à l'amplification d'un système premier, la poésie au contraire tente de retrouver une infra signification, un état pré-sémiologique du langage : bref, elle s'efforce de retransformer le signe en sens : son idéal - tendanciel - serait d'atteindre non au sens des mots, mais au sens des choses même. [...] La poésie occupe la position inverse du mythe : le mythe est un système sémiologique qui prétend se dépasser en un système factuel ; la poésie est un système sémiologique qui prétend se rétracter sur un système essentiel.»

27. Texte inédit. Nous référons pour cet article au tapuscrit daté de 2004.

28. Bill Kouélany, Extraits d'actes de naissance, tapuscrit, 2004, p. 6-7.

29. Ibid., p. 39.

30. Sa première exposition personnelle à l'Institut français de Brazzaville en 1990 est consacrée à la traduction picturale du recueil de Tchicaya U Tam'si Le mauvais sang (1955). Dans le cadre d'une exposition aux Ateliers Sahm qu'elle dirige, en 2015, ses artistes en résidence composent des œuvres plastique à partir des poèmes de Sony Labou Tansi.

31. Dans ce contexte, il s'agit des guerres civiles du Congo-Brazzaville qu'elle a traversé de 1993 à 1998.

32. Bill Kouélany, Extraits d'actes de naissance : flux et fluences, tapuscrit, 2012, p. 25. Nous respectons dans la restitution du texte les fautes de grammaire et d'orthographe qui y apparaissent.

33. Kossi Efoui, Récupérations, « Post-scriptum », Lansman, Morlanwelz (Belgique), 1992, p. 45. 